\title{
AN EXPECTED OUTBREAK OF HUMAN TRICHINELLOSIS FOR THE CONSUMPTION OF HORSEMEAT
}

\author{
TAMBURRINI A.*, SACCHINI D.** \& POZIO E.*
}

\section{Summary :}

In late February 1998, an outbreak of human trichinellosis occurred in the town of Piacenza (northern Italy) among 92 persons who had eaten raw horsemeat. The source of infection was a horse imported to the abattoir of Brescia one month previously. Although the horse had been found to be positive for trichinellosis upon routine examination, the head of an uninfected horse was exchanged with the head of the infected animal, which was mistakenly placed on the market

KEY WORDS : Trichinella spiralis, horse meat, Italy, outbreak, human infections.

In n Italy, the first outbreak of human trichinellosis due to horsemeat consumption was reported in 1975 (Mantovani et al., 1980). From 1975 to 1998 , 13 outbreaks involving 3,300 persons were described in France and Italy (Ancelle, 1998; Anonymous, 1998). At present, the consumption of raw horse meat is the most important source of human trichinellosis in Europe (Pozio, 2000). In January 1998, a naturally infected horse was detected during routine examination in a slaughterhouse in Brescia (Northern Italy). One month later, an outbreak of human trichinellosis occurred in the town of Piacenza (Emilia Romagna region, Northern Italy). The epidemiological study revealed that the two events were related.

\section{MATERIALS AND METHODS}

\section{HORSE INFECTION}

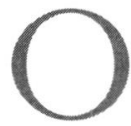
n January $22^{\text {nd }} 1998,27$ horses from Poland were imported to Italy for slaughtering. On January $26^{\text {th }}$, the routine examination carried out by the Istituto Zooprofilattico Sperimentale (IZS) showed that one of these horses was positive for trichinellosis. Larvae collected from positive muscles were counted

\footnotetext{
* Laboratory of Parasitology, Istituto Superiore di Sanità, viale Regina Elena 299, 00161 Rome, Italy.

** U.O. Malattie Infettive, AUSL, Piacenza, Italy.

Correspondence: E. Pozio, Istituto Superiore di Sanità, viale Regina Elena 299, 00161 Rome, Italy.

Tel.: +390649902304 - Fax: +390649387065 - E-mail: pozio@iss.it
}

and stored at $-80^{\circ} \mathrm{C}$ for species identification by PCR according to a published protocol (Wu et al., 1997).

\section{HUMAN INFECTION}

Serum samples were collected from 92 persons (average age 47.7 years, range $13-90$ years) with clinical signs and symptoms of Trichinella infection. All of the persons had eaten raw horsemeat purchased in the same butcher's shop (town of Piacenza). Blood samples were taken from all 92 persons, four times during the $2^{\text {nd }}, 3^{\text {rd }}, 6^{\text {th }}, 12^{\text {th }}$ and $15^{\text {th }}$ months after infection. Levels of creatine phosphokinase (CPK), lactate dehydrogenase (LDH), total IgE and eosinophilia were measured for all 92 persons. Serum samples were tested by ELISA using an ES antigen. For the four persons with severe symptomatology, a single biopsy of the deltoid muscle was performed at different points in time after infection (biopsy performed twice for one of the four).

\section{RESULTS}

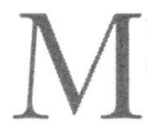

uscle tissues, collected from 38 different sites of the horse body, were highly positive for Trichinella larvae, whereas those from the horse head were negative. Veterinarians from the IZS immediately alerted their colleagues at the abattoir that the head of an uninfected horse had probably been exchanged with the head of the infected animal. The veterinarians of the abattoir ignored this warning and all of the carcasses from Poland were placed on the market except of the infected carcass with the Trichinella-negative head, which was destroyed. The artificial digestion of tissue samples showed 256 larvae per gram (lpg) in the diaphragm, $427 \mathrm{lpg}$ in the M. extensor digitorum, $381 \mathrm{lpg}$ in the $M$. biceps brachii, $312 \mathrm{lpg}$ in the M. splenius and $29 \mathrm{lpg}$ in the M. pectoralis. Larvae from horse muscles and from human biopies were identified as T. spiralis. On January $28^{\text {th }}$, the owner of a butcher shop in Piacenza (northern Italy) received four horse heads from the slaughterhouse of Brescia. The documents accompanying the four heads showed that they had originated from the stock of 27 horses imported from Poland on January $22^{\text {nd }}$. 


\begin{tabular}{lc}
\hline \multicolumn{1}{c}{ Signs and symptoms } & Infected persons (\%) \\
\hline Myalgia and fever & $92(100)$ \\
Facial oedema & $77(84)$ \\
Arthralgia & $40(43)$ \\
Gastrointestinal disorders & $39(42)$ \\
Headache & $28(30)$ \\
Haemorrhages in the conjunctivae & $5(5)$ \\
Neurological disorders & $2(2)$ \\
\hline
\end{tabular}

Table I. - Clinical features of persons infected with Trichinella spiralis.

\begin{tabular}{lcc}
\hline $\begin{array}{c}\text { Biological } \\
\text { parameters }\end{array}$ & $\begin{array}{c}\text { Infected } \\
\text { persons (\%) }\end{array}$ & Range \\
\hline Eosinophilia & $85(92)$ & $4-48.8 \%$ \\
CPK & $51(55)$ & $198-3480 \mathrm{IU}$ \\
LDH & $44(48)$ & $460-2562 \mathrm{IU}$ \\
Total IgE & $9(10)$ & $11-680 \mathrm{ng} / \mathrm{mL}$ \\
\hline
\end{tabular}

Table II. - Biological parameters of persons infected with Trichinella.

In mid-late February, 24 persons living in Piacenza were hospitalised in several wards of the local hospital for a symptomatology of unknown etiology. The other 68 infected persons had been admitted to the day hospital. All 92 persons had myalgia, fever and other symptoms (Table I); in most persons, eosinophilia, total IgE and muscular enzymes were altered (Table II). A 29-year-old woman went into a coma for one month with a severe myalgia, oedema and atrophy of the right hand muscles. Anti-Trichinella specific IgM and IgG were detected with ELISA in the serum samples of all 92 persons. All of them were treated with both mebendazole (20 mg per $\mathrm{kg}$ body weight three times daily for 10 days) and an antiinflammatory and antiallergic drug for 12 days. During treatment, all 92 persons showed prompt resolution of most signs and symptoms; however, two weeks after the end of treatment, myalgia was still present in $41(45.0 \%)$ persons, arthralgia in three $(3.2 \%)$, and fever, facial edema, headache, and neurological disorders were still present in two $(2.0 \%)$ persons. Specific IgM and IgG were also detected in all serum samples at three, six, 12 and 15 months after infection: All muscle biopsies were positive for the presence of motile larvae after artificial digestion. The number of larvae ranged from 13.4 to 36.1 per gram of muscle tissue. The mice infected with larvae from the five muscle biopsies showed Trichinella larvae in their muscles five weeks after infection.

\section{DISCUSSION}

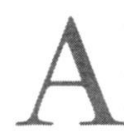

uthorities in Poland were immediately informed of the presence of an infected horse so that the farm of origin and the source of infection could be determined. Unfortunately, the name of the farmer reported on official documents was unknown in the area from which the horse was reported to have originated. In recent years, there has been a dramatic increase in the prevalence of domestic trichinellosis in former Yugoslavia, former USSR, and Romania. This epidemiological situation has certainly influenced the transmission of the infection to horses. In fact, in a period lasting less than 16 months, eight horses infected with $T$. spiralis were detected in Italy and France; however, in triangular trades, we cannot exclude that all infected horses originated from the same area, even if they arrived in the European Union from different countries.

This unfortunate episode, the first horsemeat outbreak where the source of infection was unambiguously identified, stresses the importance of correct management at abattoirs. All stages of slaughtering should be controlled and meat representing a risk to health must be properly identified and destroyed. The failure of veterinary services in France and Italy to detect Trichinella infections at the slaughterhouse has been one of the main reasons for which humans have acquired the infection through horsemeat consumption.

\section{ACKNOWLEDGEMENTS}

W e wish to thank M. Amati and F. Mancini Barbieri for their technical support. This work was made possible by the support received from the surveillance project on emerging and reemerging infectious diseases. Istituto Superiore di Sanità Art.502/12, Ministry of Health of Italy.

\section{REFERENCES}

Mantovani A., Filippini I. \& Begomi S. Indagini su una epidemia di trichinellosi umana verificatesi in Italia. Parassitologia 1980, 22, 107-132.

ANCELLE T. History of trichinellosis outbreaks linked to horse meat consumption, 1975-1998. Eurosurveillance, 1998, 3, 86-89.

Anonymous. Outbreak of trichinellosis associated with the consumption of horse meat in the Midi-Pyrénées region of France, September-October 1998. Eurosurveillance Weekly, 1998, 51, 3-5.

Pozıo E. Is horse-meat trichinellosis an emerging disease in EU? Parasitology Today, 2000, 16, 266.

Wu Z., Nagano I., Fukumoto S., SaIto S., Yamaguchi T., Pozio E. \& Takahaschi Y. Polymerase chain reaction primers to identify Trichinella spiralis or Trichinella pseudospiralis. Parasitology International, 1997, 46, 149-154. 